Questions vives

\section{Questions Vives}

Recherches en éducation

$\mathbf{N}^{\circ} 24 \mid 2015$

Accompagnement des transitions professionnelles et dispositifs réflexifs en formation initiale et continue

\title{
Accompagner la mastérisation de la formation des enseignants en France : un dispositif de régulation pour les formateurs des IUFM
}

Accompany the inscription of the training of the teachers in Master's degree in France: a device of regulation for the trainers of French Schools of Education

\section{Vincent Grosstephan}

\section{(2) OpenEdition}

\section{Journals}

Édition électronique

URL : http://journals.openedition.org/questionsvives/1786

DOI : 10.4000/questionsvives. 1786

ISSN : 1775-433X

Éditeur

Université Aix-Marseille (AMU)

Édition imprimée

Date de publication : 15 décembre 2015

ISBN : 978-2-912643-48-3

ISSN : 1635-4079

Référence électronique

Vincent Grosstephan, « Accompagner la mastérisation de la formation des enseignants en France : un dispositif de régulation pour les formateurs des IUFM », Questions Vives [En ligne], № 24 | 2015, mis en ligne le 15 février 2016, consulté le 19 avril 2019. URL : http://journals.openedition.org/ questionsvives/1786; DOI : 10.4000/questionsvives.1786

Ce document a été généré automatiquement le 19 avril 2019.

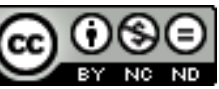

Questions Vives est mis à disposition selon les termes de la licence Creative Commons Attribution -

Pas d'Utilisation Commerciale - Pas de Modification 4.0 International. 


\title{
Accompagner la mastérisation de la formation des enseignants en France: un dispositif de régulation pour les formateurs des IUFM
}

\author{
Accompany the inscription of the training of the teachers in Master's degree in \\ France: a device of regulation for the trainers of French Schools of Education
}

\author{
Vincent Grosstephan
}

\section{Introduction}

1 Depuis une vingtaine d'années, sous les effets d'une volonté de professionnalisation de la formation des enseignants, les formateurs ${ }^{1}$ sont confrontés à de multiples évolutions de leur cadre de travail. Plus récemment, l'intégration des IUFM dans les universités (2008), puis la réforme de la formation des enseignants, dite de «la mastérisation " (2010) participent d'un processus d'universitarisation (Bourdoncle, 2007) de la formation aux métiers de l'enseignement, processus poursuivi dans le cadre de la création des Écoles Supérieures du Professorat et de l'Éducation (2013). Ce passage d'une formation des enseignants dispensée soit dans le cadre des Écoles Normales pour les professeurs des écoles ou dans les Centres Pédagogiques Régionaux pour les enseignants du second degré à une formation dans le cadre d'un diplôme universitaire ${ }^{2}$ se traduit par " une redéfinition plus ou moins importante du travail des formateurs" (Perez-Roux, 2012, p. 40). Si la formation des enseignants du second degré s'est toujours appuyée sur une forte dimension universitaire, ce processus d'universitarisation est nouveau pour la formation des professeurs des écoles. On peut, selon Bourdoncle (2007), dire que dans les IUFM, ce processus confronte les formateurs à deux évolutions majeures. D’une part, « les savoirs y sont désormais transmis, mais aussi créés et accumulés selon les règles particulières de l'université faisant une large place à l'activité de recherche » (Bourdoncle, 2007, p. 138), 
et, d'autre part, un statut professionnel devient «dominant, celui d'enseignantchercheur » (p. 138). Dans les IUFM, entre la logique d'universitarisation et la logique de professionnalisation, se «dessine un nouveau paysage de la formation initiale et continue » (Perez-Roux, 2012, p. 41). De nouvelles modalités de travail se développent, de nouveaux dispositifs sont mis en place, les formateurs sont confrontés à un nouveau public, «les étudiants». Ainsi, dans le quotidien de leur travail les formateurs doivent faire face à des situations de travail nouvelles pour eux et pour les étudiants. Ils doivent donc « intégrer les nouvelles modalités de la réforme, les mettre en œuvre sur le terrain et travailler avec d'autres composantes de l'université» (p. 41), autant d'éléments qui ne sont pas sans susciter des débats, des interrogations au sein des IUFM. Ces débats et interrogations portent, pour partie, sur la place de la recherche dans une formation à visée professionnalisante, sur la nature et la légitimité d'une « initiation à la recherche »; sur la nature même des enseignements, mais ils questionnent également la professionnalité, l'identité, la légitimité et le sens du travail des formateurs (Perez-Roux, 2012) : formateurs ou enseignants pour reprendre la distinction de Charlot (1990) ? Comme l'évoque Perez-Roux en introduction de ce numéro thématique, cela interroge de fait les choix qu'ils sont amenés à faire dans cette situation de transition professionnelle au sein d'un environnement changeant et générateur de déséquilibres, de déstabilisations voire de rupture identitaire (Dubar, 1992; Ricoeur, 1990). Va-t-on y observer le déclenchement d'un épisode de développement, les acteurs se saisissant du contexte pour en faire une occasion et un moteur d'évolution professionnelle et personnelle ? Ou verra$\mathrm{t}$-on un processus régressif, de déprofessionnalisation d'une partie des formateurs, les plus éloignés des logiques et normes universitaires (Demailly \& De La Broise, 2009) ? C'est à ces questions que cet article tentera de répondre dans un premier temps à partir d'une analyse du point de vue exprimé par les formateurs. Nous tenterons de montrer en quoi les logiques institutionnelles, structurelles et relationnelles déterminent et «affectent » les acteurs, générant émotions et affects (Lordon, 2013). Loin d'une perspective psychologisante, nous tenterons d'aborder ce moment particulier de transition dans le cadre d'une «structure peuplée d'individus conçus comme des pôles de puissance désirante, dont le désir, précisément, peut parfois aspirer à échapper aux normalisations et, dans certaines conditions y parvenir » (Lordon, 2013, p. 12). Puis il tentera, dans un second temps, de montrer qu'un dispositif réflexif fondé sur l'analyse des situations de travail et de l'activité qui y est déployée est susceptible de redonner la main aux formateurs sur leur métier.

2 Les résultats d'une récente enquête de Thérèse Perez-Roux (2012) réalisée à partir d'un questionnaire proposé à l'ensemble des IUFM apportent de premiers éléments de réponse à ce questionnement. Il apparaît que les mutations institutionnelles relatives à la mastérisation de la formation aux métiers de l'enseignement sont, pour $82 \%$ des formateurs qui ont répondu au questionnaire, perçues et vécues comme rendant plus difficile l'exercice du métier. Toutefois, la perception de ces évolutions est très contrastée, ainsi $59 \%$ des formateurs mettent en avant des aspects positifs. Au moment de la création en septembre 2013 des ESPE, ces résultats témoignent d'un «malaise des formateurs » à l'égard de leur travail en IUFM. Il semble donc nécessaire, pour prolonger cette enquête, de chercher à mieux comprendre ce qu'est être formateur en IUFM/ESPE 3 ? 


\section{Cadre conceptuel}

\subsection{La professionnalisation de la formation des enseignants et des formateurs en France : quels enjeux?}

Nous considérons avec Lang (2001) que «la notion de professionnalisation est un des thèmes essentiels de la rhétorique de la réforme de l'école d'aujourd'hui ». En effet, il n'est pas de débat sur la formation des enseignants qui échappe à l'emprise de cette notion, tant d'un point de vue institutionnel (du rapport De Peretti en 1982 à la réforme de la formation des enseignants de 2013 et la création des ESPE) que du point de vue de la recherche en éducation (des travaux initiaux de Schön, 1994, aux travaux en analyse ergonomique de l'activité des enseignants (Amigues, 2003 ; Saujat, 2004) ou en didactique professionnelle (Pastré, 2002; Vinatier, 2009) par exemple). Pour autant la professionnalisation comme horizon pour les enseignants français recèle autant de difficultés et d'obstacles que d'espoirs (Bourdoncle, 1993; Maroy, 2006). Les deux difficultés qui selon Bourdoncle (p. 104) «touchent au cœur du processus de professionnalisation : la nature des savoirs et la collaboration des instances concernées, l'école, lieu de la pratique professionnelle et l'université, lieu de la formation » restent d'une brulante actualité au regard des débats relatifs à la " mastérisation » et plus encore à la création des ESPE où la répartition des interventions entre universitaires et professionnels de l'enseignement est source de tensions. Ces difficultés et tensions se répercutent in fine sur l'activité professionnelle des personnes chargées de professionnaliser les enseignants : les formateurs d'IUFM hier, d'ESPE aujourd'hui. Quid de leur propre professionnalisation? Bien qu'incertaine et non acquise (Altet, Paquay \& Perrenoud, 2002) il semble bien que les réformes successives d'universitarisation de la formation nous installent dans une période clé de ce processus où «les stratégies, les institutions, les acteurs » seront sans doute décisifs.

\subsection{Enseigner en IUFM/ESPE : une approche ergonomique du travail de formateur}

\subsubsection{Une double visée épistémique et transformative}

4 Aborder le travail du formateur en IUFM/ESPE à partir d'une référence ergonomique c'est tenter, d'une part, d'accéder à une compréhension la plus fine possible de l'activité déployée par les acteurs dans des situations de travail données, et d'autre part, d'agir sur ces situations de travail et sur le pouvoir d'agir des formateurs pour adapter le travail à l'homme et rendre compatibles la préservation de leur santé avec les finalités de l'institution qui les emploie (Ria, 2008). Cette double visée épistémique et transformative, caractéristique de l'ergonomie francophone, est au cœur du dispositif que nous proposons d'étudier ici.

- Une visée épistémique : comprendre le travail du formateur en IUFM/ESPE. Nous proposons une entrée par les situations de travail (Mayen, Métral \& Tourmen, 2010) dans la mesure où nous considérons, avec ces auteurs, qu'il est nécessaire, pour comprendre, décrire, analyser l'activité des formateurs ainsi que les ressources dont ils peuvent disposer, d'identifier et de caractériser au préalable les situations de travail dans lesquelles les formateurs sont plongés. Cette visée épistémique d'analyse du travail s'appuie ainsi plus particulièrement 
sur les distinctions qui fondent l'approche ergonomique. Il s'agit tout d'abord de la distinction entre la tâche prescrite et l'activité mobilisée par le formateur confronté à cette tâche. Il s'agit ensuite de la distinction entre l'activité prescrite et l'activité effectivement réalisée. Il s'agit enfin de la distinction entre l'activité réalisée et le réel de l'activité (Clot, 2008), cette dernière intégrant les dimensions non visibles de l'activité comme les empêchements, les activités contrariées ou les dilemmes que le formateur a eu à gérer.

- Une visée transformative. Il s'agit ici tout d'abord de permettre aux formateurs de s'emparer de cette analyse des situations de travail et de l'activité déployée pour définir les possibilités d'un développement du pouvoir d'agir et d'élargissement de la prise en main personnelle et collective de leur métier (Roger, 2007). Il s'agit ensuite de se servir des résultats produits par l'analyse du travail des formateurs pour interpeler la direction quant à la politique d'accompagnement des transitions professionnelles et à l'organisation du travail au sein de l'IUFM.

\subsubsection{Les déterminants de l'activité des formateurs}

Dans la perspective ergonomique que nous avons adoptée, nous faisons nôtre le point de vue qui « consiste à affirmer que les principes de l'action des [formateurs] ne résident pas uniquement dans la situation où se déroule l'interaction, mais qu'il faut les rechercher aussi dans les prescriptions et dans l'activité d'interprétation et de redéfinition à laquelle elles donnent lieu de la part des [formateurs] ${ }^{4}$ » (Saujat, 2002, p. 63). C'est donc au croisement des conditions concrètes de l'action, notamment de l'action conjointe avec d'autres professionnels ou les étudiants, et des multiples déterminants institutionnels, collectifs et personnels que s'actualise l'activité réelle des formateurs. Notre démarche consiste à essayer de percevoir le poids et le rôle de ces déterminants à partir d'une analyse du réel de l'activité au sein des situations professionnelles que nous aurons préalablement caractérisées. En effet, et bien qu'ayant considéré plus haut que les déterminants de l'action ne résident pas uniquement dans la situation, avec Mayen, Métral et Tourmen (2010, p. 32), nous pensons que les « situations sont premières parce que ce sont elles dont les professionnels ont, ou auront, à se débrouiller dans le cours de leur vie professionnelle ». Nous considérons que l'identification et la caractérisation des situations de travail en tant que système de contraintes imposées à l'activité des professionnels est un préalable nécessaire à l'analyse de cette activité ainsi que des ressources nécessaires pour penser et agir. En didactique professionnelle, «un professionnel compétent est une personne capable de maîtriser un ensemble de situations professionnelles, et, par extension, de classes de situations, plus ou moins complexes, constitutives de son emploi ou métier " (Leplat, 1991; Vergnaud, 1990, 1996; Pastré, 2004 ; Ferron et al. 2006). Ces situations possèdent une double dimension impactant fortement les compétences des acteurs: une dimension générique, chaque situation comportant un certain nombre de traits qui l'assimilent aux autres situations d'une même classe et qui permettent une orientation et une organisation de l'action (cf. théorie des schèmes, Vergnaud, 1996), et une dimension singulière, située. Identifier les parts de générique et de singulier dans les situations de travail des formateurs devient dès lors un enjeu essentiel pour l'analyse du travail. 


\section{Problématique et questions de recherche}

6 Il s'agit d'analyser le travail, d'une part en identifiant et caractérisant les situations de travail et d'autre part en étudiant l'activité (y compris dans ses dimensions contrariées) mobilisée par les professionnels engagés dans ces situations qui y vivent ce que Maleyrot (2013) appelle des épreuves professionnelles conditionnelles et situationnelles ${ }^{5}$. La réforme de la mastérisation et ses conséquences concrètes sur l'activité des formateurs engagés dans des situations de travail parfois fortement transformées expriment de façon archétypale ces deux types d'épreuves.

7 La première question à laquelle nous tenterons dès lors de répondre est la suivante : de quelle(s) façon(s) ces déterminants institutionnels, structurels et relationnels « affectentils $»^{6}$ les formateurs des IUFM?

8 La seconde question interroge le dispositif réflexif tel qu'il est proposé dans cette étude et notamment ce en quoi il est susceptible de permettre aux acteurs de se réapproprier individuellement et collectivement la «renormalisation» (Schwartz, 2012) de leur activité professionnelle dans ces contextes institutionnels et structurels. Néanmoins, la réponse à cette seconde question ne sera qu'esquissée de façon prospective, les données recueillies pour cette étude ne permettant pas de documenter réellement la réponse à cette question.

\section{Méthodologie}

9 L'étude porte sur l'analyse de séminaires de formation dans un IUFM. Nous avons décidé d'organiser au plus près des lieux de travail (les cinq sites de l'académie) des séminaires visant à donner la parole à tous les formateurs volontaires quant aux conséquences sur leur activité quotidienne de cette réforme de la "mastérisation». Ces séminaires ne constituent ni un moyen de contrôle normatif ni une tribune revendicative (même si de toute évidence émergent des demandes, des propositions, mais que nous voulons appuyées sur une analyse fine de l'activité professionnelle). Ils visent, par une méthodologie rigoureuse, à investiguer le réel de leur activité au sens de Clot (2008), c'est à dire à s'intéresser avant toute chose à ce qui les préoccupe, aux activités contrariées, empêchées, aux dilemmes et difficultés auxquels ils sont confrontés dans l'exercice de leur métier.

\subsection{Recueil de données}

Les données recueillies au cours des trois phases qui ont structuré les séminaires sont issues des verbalisations des acteurs au cours des échanges lors des séminaires. Ces échanges ont fait l'objet d'enregistrements audio après accord préalable des participants.

La première phase du séminaire consiste à identifier les situations et classes de situations de travail auxquelles sont confrontés les formateurs d'IUFM. Ce premier travail a également pour but d'identifier quelles sont les situations de travail largement partagées par les acteurs et celles très spécifiques. Nous pensons en effet que le sentiment d'appartenance à une communauté de professionnels est fortement lié à la possibilité de se reconnaître dans des pratiques partagées. 

travail d'avant la "mastérisation» et celles consécutives à la réforme. Au-delà de la nature même des situations qui pourrait être amenée à évoluer, il s'agit également ici de repérer des situations nouvelles, inédites, ou la disparition d'autres situations de travail. Pour cela, il nous semble indispensable de dépasser les analyses des traits de surface et la dénomination des situations en décrivant le plus précisément possible les actions habituellement réalisées dans ces situations de travail.

La dernière phase a pour but d'aller au-delà de la simple description de la partie visible et observable de l'activité en tentant d'identifier les difficultés, dilemmes, obstacles rencontrés dans son exercice quotidien. Nous considérons avec Clot $(1999,2008,2010)$ que l'action réalisée n'est en fait que celle qui a "gagné " parmi toutes celles possibles. L'acteur est parfois amené à renoncer à certaines actions ou à voir les actions qu'il a choisies contrariées pour diverses raisons: sentiment d'incompétence, absence de ressources institutionnelles et/ou collectives de l'action etc. Ce sont ces facettes de l'activité empêchée, contrariée que nous souhaitons enfin faire émerger lors de ces séminaires.

\subsection{Traitement des données}

14 Les échanges entre les participants aux séminaires sont ensuite intégralement transcrits et font l'objet d'une analyse en trois temps correspondant aux trois phases structurant le déroulement des séminaires :

15 Un premier temps consiste à catégoriser les situations de travail identifiées par les acteurs en classes de situations. Ce travail de catégorisation est réalisé collectivement et validé par les participants lors de chaque séminaire. Une synthèse des cinq séminaires est ensuite réalisée par le chercheur ;

16 Un second temps vise à inférer de l'identification et de la description des actions habituellement réalisées dans ces situations l'évolution des situations lors du passage à une formation «mastérisée ». Nous avons procédé ici à une analyse de contenu (Bardin, 1991) de type inductif, visant à faire émerger des discours des participants leur perception des principales caractéristiques d'évolution des situations de travail ;

Un troisième temps vise enfin à caractériser les dimensions non visibles de l'activité et les significations que les acteurs attribuent aux situations vécues, mobilisant en cela les grilles de lecture de la clinique de l'activité. Il s'agit ici d'extraire des discours des acteurs les préoccupations dont ils font état et qui renvoient à ce que le passage à la mastérisation a induit comme reconfiguration de leur activité. L'extraction des données relatives à ces éléments a ensuite donné lieu à une catégorisation réalisée par le chercheur.

\section{Résultats}

Au-delà de quelques aspects spécifiques aux différents sites (présence ou non de différentes spécialités de master, plus ou moins forte présence d'enseignants-chercheurs, plus ou moins grand éloignement géographique du site principal), les constats que nous avons pu réaliser lors des cinq séminaires témoignent de bouleversements et de 
processus d'adaptation largement partagés par les 53 formateurs ayant participé à ces séminaires, quel que soit le lieu.

\subsection{Des déplacements des objets de l'activité : un processus de fragmentation, d'émiettement dû aux contraintes universitaires}

19 Nous regroupons dans cette partie l'analyse des deux premiers temps du dispositif (catégorisation des situations de travail et analyse des évolutions perçues par les acteurs). L'identification des situations de travail nous a ainsi conduits à proposer une catégorisation en onze classes de situations de travail ${ }^{7}$.

Néanmoins, l'interrogation sur les dimensions de changement et de continuité des situations de travail, l'analyse des caractéristiques de ces dernières, la description des actions habituellement réalisées dans ces situations (temps 2) montrent d'une part un réel processus de complexification et de diversification (que nous ne développerons ici), d'autre part d'importants déplacements dont nous présentons ici les trois principaux.

\subsubsection{Un déplacement d'activité : d'une activité de formation à une activité d'enseignement...}

21 Le processus de fragmentation et d'émiettement a été très précisément décrit dans tous les sites et constitue, pour les formateurs de l'IUFM, une conséquence majeure de l'organisation du diplôme en $\mathrm{UE}^{8}$ et $\mathrm{EC}^{9}$. Il se caractérise tout d'abord par un déplacement des objets de l'activité :

Le premier déplacement concerne ce que certains formateurs ont nommé une "scission » entre l'enseignement du master et la formation sur le terrain. Cette scission est vécue comme un manque essentiel. Elle s'avère d'autant plus inévitable que le temps des enseignements universitaires n'est pas le temps de la formation professionnelle, qui, de l'avis des formateurs, est considérablement réduite par rapport au système de formation antérieur, comme l'évoque à regret ce formateur: «il n'y a presque plus de stages, et après ils sont balancés sur le terrain direct, sans véritable accompagnement. Or des compétences professionnelles ça met du temps à se construire. Mais dans les masters on a l'impression que ce n'est plus notre rôle ». Il est à noter ici que les formateurs de l'IUFM n'intègrent pas dans leur analyse l'année de stage ${ }^{10}$ mais comparent PLC1/PE1-PLC2/PE2 ${ }^{11}$ et $\mathrm{M} 1-\mathrm{M} 2^{12}$, considérant que l'année de stage, vues ses conditions de mise en œuvre, ne peut être assimilée à une véritable année de formation. Ces perceptions largement partagées par les acteurs appellent un certain nombre de questions qu'il conviendrait d'approfondir, notamment en ce qui concerne les critères permettant de distinguer ce qui relève d'enseignements universitaires et ce qui relève d'une formation dite professionnelle. Les enseignements universitaires ne seraient-ils pas professionnalisants? Les éléments de formation professionnelle seraient-ils ciblés sur les UE relatives aux stages? Autant d'éléments qui questionnent la cohérence globale du diplôme et sa mission de professionnalisation.

23 Les formateurs de l'IUFM considèrent en outre que le recentrage disciplinaire induit par l'organisation en UE et EC aboutit à une spécialisation, facteur d'éparpillement et d'émiettement des enseignements. Cet enseignant considère par exemple que : « on fait des cours de plus en plus pointus, sur des thématiques très ciblées mais aussi très réductrices par rapport aux vraies questions du métier. Chacun fait son petit truc dans 
son coin sans savoir ce que font les autres. Du coup on n'arrive plus à faire de liens ». Or ils n'identifient pas non plus d'instance ou de lieu de coordination et de mise en cohérence de l'ensemble des enseignements. Cette cohérence relève avant tout du travail d'élaboration de la maquette: «on a bien essayé de construire de la cohérence avec la maquette, mais après c'est chacun sa partie et chaque partie est un tout petit bout d'un tout sans que des liens soient faits ensuite ». Cet important travail initial a été suivi d'une mise en œuvre selon une logique de partage des tâches (confiée notamment aux responsables d'EC). Cette mise en œuvre n'a pu, pour des raisons essentiellement liées à la charge de travail et à la complexité du dispositif (notamment les exigences d'harmonisation inter-sites), selon les formateurs, être accompagnée d'une réflexion sur la réelle cohérence de l'ensemble: «une fois la maquette conçue, on a la tête dans le guidon et on n'a plus le temps de voir ensemble si ce qu'on fait est cohérent ».

Cet émiettement semble également induit par la modalité «cours magistral » perçue comme dominante - bien qu'une analyse objective de la maquette fait état d'une majorité de travaux dirigés (TD) ou travaux pratiques (TP) - qui ne permettrait plus des éclairages multiples et/ou croisés sur des situations professionnelles mais supposerait des enseignements plus disciplinaires et plus spécialisés : « les étudiants ont maintenant une majorité de cours magistraux (CM). Du coup ils ont des contenus plus spécialisés mais pas de lieu pour les confronter autour de questions professionnelles ». De fait, un paradoxe est évoqué à plusieurs reprises par les formateurs: le niveau académique des enseignements s'est élevé (plus pointu, plus étayé par des savoirs considérés comme légitimes, produits par la recherche) mais le niveau d'appropriation baisserait en raison de la perte de sens des contenus enseignés eu égard à l'absence d'ancrage dans les pratiques professionnelles vécues par les étudiants : «ah c'est sûr que les cours sont plus pointus, mais j'ai l'impression que les étudiants n'y trouvent plus de sens. On leur balance des contenus mais ils ne comprennent pas à quoi ça sert. Dans quelle situation professionnelle ça va leur servir». Les savoirs en tant que tels ne sont pas remis en question (certains y voient même un gage de reconnaissance pour la formation ou y trouvent un plaisir renouvelé du fait de l'élévation du niveau des contenus enseignés). Par contre, la modalité « cours magistral », l'absence de ponts entre enseignements et la perte de l'ancrage dans des pratiques professionnelles vécues les rendraient moins pertinents, moins efficaces: «en fait, c'est pas que ces contenus sont inutiles mais en utilisant essentiellement les $\mathrm{CM}$ on n'arrive plus vraiment à faire des ponts avec leurs pratiques de classe, quand ils en ont... ». D'autres formateurs y voient aussi, pour les mêmes raisons que celles évoquées précédemment mais également en raison des origines diverses des étudiants, plus une initiation qu'un affermissement des contenus car la recherche de l'essentiel, dans un temps considéré comme trop court, produit parallèlement la superficialité des acquisitions : " plus on réduit le temps disponible plus tu fais les choses à moitié, tu restes à la surface des choses. Tu n'as pas le temps d'approfondir alors que certains étudiants en ont vraiment besoin en fonction de ce qu'ils ont fait avant de venir à l'IUFM ».

Par ailleurs, les formateurs voient comme conséquences de cette fragmentation, l'impossibilité d'un réel suivi des étudiants mais aussi, pour certaines disciplines (EPS, musique), un appauvrissement des contenus par le jeu des options : "on a déplacé les cours obligatoires en EPS vers des options, du coup beaucoup d'étudiants auront une formation très superficielle dans la discipline qu'ils devront pourtant enseigner après ». D'autres enfin regrettent la disparition des situations d'analyse de pratiques rendues 
quasi-caduques par la distance au terrain devenue très importante et la modalité dominante d'enseignement (magistrale et disciplinaire) comme l'évoque cette autre enseignante : «c'était (les analyses de pratiques) vraiment ce qui faisait notre spécificité de formation professionnelle. De toutes façons il n'y a presque plus de stage. Donc tu fais une formation théorique, tu fais des cours, en plus surtout des cours magistraux, tu ne fais plus de la formation ».

Ainsi, l'inscription de la formation des enseignants dans le cadre d'un diplôme universitaire fait dire aux formateurs qu'ils ont le sentiment d'être passés d'un statut de formateur à un statut d'enseignant : « [...] avant je me pensais formateur. Aujourd'hui j'ai l'impression de ne faire que des cours ». " On enseigne un savoir, on forme un individu » disait Charlot (1990, p. 6). En d'autres termes, leur activité se serait déplacée d'un objectif de développement de compétences professionnelles vers un objectif de transmission de savoirs comme l'exprime cette enseignante: «je suis venue à l'IUFM pour former des jeunes au métier d'enseignant. Là je fais des cours sur l'enseignement ou parfois même sur autre chose mais je ne les aide plus à développer des compétences professionnelles ». En caractérisant la logique de formation par la référence aux pratiques, «par définition finalisées et contextualisées»(p.7), par une activité du formateur centrée sur la médiation, la prise en compte de parcours et trajectoires individuelles, et la logique de l'enseignement par l'élaboration et la diffusion de discours constitués, de concepts, d'acquis cumulés en patrimoine culturel, Charlot met l'accent sur des différences sensibles de l'activité déployée dans les deux cas. Deux types d'acteurs se reconnaissent tout particulièrement dans ces deux types d'activité : les formateurs issus du premier ou second degré dont l'identité penche du côté de la formation et les enseignants-chercheurs qui tirent leur légitimité des savoirs de haut-niveau dont ils sont dépositaires. De fait, en renvoyant l'essentiel de la professionnalisation et de la référence aux pratiques professionnelles à l'après-master, la réforme renforce la place des enseignantschercheurs au sein des diplômes, au détriment des formateurs de statut premier ou second degré.

\subsubsection{Un déplacement d'objets de l'activité de suivi : d'un suivi du parcours de professionnalisation à un suivi centré sur des éléments de diplôme disparates...}

L'analyse des discours relatifs à cette classe de situations montre un déplacement des objets de suivi : d'un suivi centré sur le développement de compétences professionnelles et le parcours de formation à un suivi ciblé sur des éléments de diplôme (mémoire d'initiation à la recherche, Certificat Informatique et Internet niveau 2 Enseignant (C2inv2e), travaux disciplinaires notamment) qui amènent à une nouvelle répartition des tâches faisant plus qu'auparavant appel à des spécialistes : "maintenant tu ne suis plus un étudiant, tu suis du C2i, tu suis du mémoire, tu suis des travaux particuliers, et c'est chacun dans son domaine de spécialité ». Les formateurs regrettent pour un grand nombre d'entre eux la quasi-disparition du suivi de stage que la «mastérisation » a délégué aux maîtres-formateurs et conseillers pédagogiques ainsi que des équipes pluricatégorielles de suivi : « là on a beaucoup perdu avec le suivi par des équipes de suivi. Ça c'était bien, on pouvait croiser les points de vue et vraiment apporter quelque chose de complémentaire au stagiaire ». Le suivi vise avant toute chose à s'assurer de la maitrise des savoirs et concepts propres à chaque $\mathrm{EC}$ du master et non plus à accompagner la construction de la professionnalité des étudiants. ${ }^{13}$ 


\subsubsection{Les déplacements de l'activité de concertation.}

absolument nécessaire mais en même temps extrêmement difficile. Cette difficulté semble trouver ses racines dans un processus paradoxal de décentralisation et de recentralisation, tout particulièrement sensible dans les tensions entre des injonctions $\mathrm{d}$ 'harmonisation académique et les logiques propres de site, entre la logique propre du master et l'articulation avec des interventions de partenaires extérieurs. La concertation interne au master porte sur l'harmonisation des contenus d'enseignement au sein des départements, avec les responsables d'EC, sur la conception des sujets d'examens et des modalités d'évaluation (critères et indicateurs d'évaluation), sur la concertation relative à l'organisation (emploi du temps, déplacements etc.). La concertation avec les partenaires extérieurs, quant à elle, porte tout particulièrement sur l'organisation des stages (Inspection d'Académie / Rectorat; directeurs d'école; maîtres-formateurs / maîtres d'accueil temporaire; responsables d'associations ou autres organismes...), sur les interventions en formation continue (Inspection Académique / Rectorat), sur les interventions dans d'autres établissements (Institut de Formation en Soins Infirmiers...) ou composantes de l'université, sur l'intervention de personnes extérieures à l'université ou à la composante. quant à ces activités de coordination qui constituent à leurs yeux une part très importante de leur charge de travail. Ce travail de coordination est caractérisé par les formateurs par un certain nombre de mouvements forts :

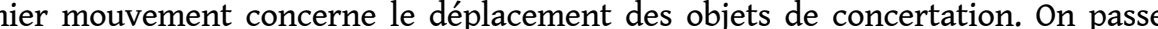
d'une concertation à propos des personnes et de leur professionnalité (équipes de suivi de PE2 notamment) à une concertation à propos des contenus disciplinaires : "avec le master on se concerte pour les cours et plus pour suivre les étudiants. D'ailleurs on ne parle plus des étudiants mais que des contenus de cours ». Ce mouvement s'exprime par ailleurs dans le sentiment qu'ont beaucoup de formateurs de passer d'un métier de formateur où l'on professionnalise des individus à un métier d'enseignant où l'on délivre des savoirs.

31 Ce mouvement induit un changement d'échelon de la coordination. L'échelon est moins local et plus académique. Les formateurs ressentent fortement l'injonction d'harmonisation académique justifiée par la nécessité de délivrer le même diplôme sur les différents sites et donc de faire en sorte que les contenus délivrés soient les mêmes. Ils voient dans la logique multi-sites moins un processus de décentralisation qu'un processus de diffusion contrôlée centralement. Une autre facette en est le cloisonnement des concertations. Certains formateurs considèrent qu'il n'y a pas moins de concertation qu'avant mais une reconfiguration des groupes au sein desquels s'opère cette concertation. Les groupes sont plus disciplinaires, plus académiques et moins locaux. Une des instances de concertation sur les contenus est le département disciplinaire. Une autre instance est l'équipe pédagogique d'EC ${ }^{14}$. Cette injonction à l'harmonisation des contenus et son corollaire, la concertation académique, ont pour conséquence chez certains formateurs, le sentiment d'un processus de dépossession de son activité de conception de cours. L'un d'eux se pose par exemple la question suivante : « est-ce que je suis à l'origine de mes cours? " évoquant le choix de son département de proposer des cours à reproduire à l'identique sur les différents sites. Cette prescription assimile d'une certaine 
façon l'activité d'enseignement à une simple activité d'exécution. La présence de cette prescription se retrouve d'ailleurs dans deux cas de figure différents, évoqués par les formateurs.

Le premier relève d'une prescription où le spécialiste de la question dans le département réalise le cours ainsi que son support (un diaporama) que les autres formateurs concernés doivent reproduire tel quel : « en fait tu ne fais qu'appliquer le cours qu'on te donne. Tu ne choisis rien : tu appliques ». Cette prescription remontante (Six, 1999) repose le plus souvent, aux dires des formateurs, sur des hiérarchies implicites liées au statut des prescripteurs («c'est le PU [professeur des universités] qui donne le cours. C'est lui qui sait. Nous on n'a le droit que d'appliquer»). On peut y voir, dans ce contexte de mastérisation, une forme de reconstitution d'une hiérarchie universitaire dont les EC ne pouvaient se prévaloir du temps d'avant la mastérisation.

Le second relève d'une autre forme de prescription remontante provenant du collectif de travail. Des formateurs ont relaté l'intense travail collectif à l'origine des cours qu'ils avaient à dispenser aux étudiants. La concertation a été telle qu'ils ont eu le sentiment d'une quasi-fusion avec leurs collègues doublé d'un malaise quant à leur propre individualité. Ils ont avoué respirer quand ils ont su mettre de leur personne dans le cours et prendre quelques distances avec la prescription.

Nous avons ici deux exemples de la façon dont les formateurs se sont adaptés à l'exigence d'harmonisation, adaptation d'abord source d'insatisfaction avant de s'autoriser à prendre plus de distance avec les prescriptions.

La structure a choisi, pour faciliter l'harmonisation des contenus d'UE et d'EC, une organisation multipliant les responsabilités : responsable de mention, de spécialité, de parcours, d'UE, d'EC, de stage, DE (directeur des études), de département disciplinaire... au point que rares sont les formateurs rencontrés qui n'exercent pas l'une ou l'autre d'entre elles. Certains y voient un double mouvement de fragmentation et de dilution de la responsabilité : « en fait tout le monde est responsable d'un petit quelque chose mais au bout du compte qui est responsable de l'ensemble? On ne sait plus trop qui fait quoi et on n'a plus de véritable lisibilité de ce qui se fait ». Ils suggèrent une définition plus précise des périmètres d'action et de décision de ces responsables ainsi qu'une réflexion sur l'ensemble de l'organigramme, craignant que la multiplication des responsabilités ne rende la vision d'ensemble de plus en plus difficile pour tout le monde.

Ces sentiments apparaissent ainsi quelque peu paradoxaux dans la mesure où ils associent la perception d'une augmentation significative de la diversité et de la complexité des situations de travail par rapport à la période d'avant la réforme à l'impression de recentrage sur les contenus disciplinaires et de spécialisation des intervenants où les formateurs de statut premier et second avouent avoir du mal à trouver leur place : « on a du mal à trouver notre place. Les cours pointus c'est les universitaires. Nous on prend ce qu'il reste ou ce qu'ils ne veulent pas faire ».

\subsection{Des caractéristiques des situations de travail au travail réel : état des préoccupations des acteurs}

\subsubsection{Une activité empêchée, contrariée}

37 Au regard de ces divers déplacements, l'activité est souvent ressentie comme une activité contrariée voire empêchée. Les formateurs ont ainsi évoqué à plusieurs reprises 
l'impossibilité pour eux de s'inscrire (sauf cas particuliers) dans une démarche d'évaluation formative : "on fait en même temps entraînement et notation » (référence aux contrôles continus où le travail d'évaluation se superpose au travail d'apprentissage alors que le premier n'accorde pas la même place à l'erreur que le second) ce qui constitue un véritable conflit de logiques. La pression évaluative semble ainsi constituer un empêchement majeur à cette activité formative que les formateurs évoquent à propos des années PE2 ou PLC2: «on a l'impression de noter tout le temps et pour tout ». Par exemple, « les stratégies d'étudiants ayant un cursus en mathématiques qui consistent à choisir l'option mathématiques pour "assurer" une bonne note plutôt que de combler leur manque en français » en sont très représentatives. Cela peut également être amené à sous-estimer le poids dont disposait l'évaluation en PE2 / PLC2, qui, bien que portant sur d'autres objets (les compétences professionnelles) n'en était pas moins prégnante (BrauAntony \& Jourdain, 2004). On retrouve trace d'activités contrariées lors de l'élaboration des sujets d'examens : il y a une tension forte entre la volonté de proposer des sujets de master déconnectés des exigences des concours tout en voulant qu'ils servent aux étudiants à... réussir les épreuves du concours !: «On (la direction mais aussi la prescription institutionnelle qui demande à bien dissocier les exigences du master et la préparation au concours) nous demande de faire des sujets qui ne sont pas préparation au concours. Alors, on essaie de trouver des sujets neutres mais qui peuvent quand même les aider pour le concours. C'est un exercice particulier». Sont évoquées également des tensions entre des exigences plus globalisantes et professionnelles du concours et des exigences plus disciplinaires et parcellaires des examens de master; entre des attentes méthodologiques et des attentes académiques, savantes.

\subsubsection{Des stratégies dites « de contournement » et « de compensation »}

Confrontés à ce sentiment de fragmentation des enseignements et au constat des difficultés des étudiants, les formateurs mettent en œuvre ce qu'ils appellent des stratégies de contournement et de compensation notamment par du conseil bénévole (qu'ils appellent " conseil off ») ou la mise en ligne de cours ou de compléments de cours. La perception de ces actions auprès des étudiants comme relevant d'une compensation questionne les prescriptions en la matière. Peut-on effectivement considérer ce travail de " conseil off» ou de mise en ligne de cours comme de la compensation ou ne doit-on y voir qu'une part "normale» de l'activité des formateurs, qui s'inscrirait dans ses missions? Cette question s'avère source de tensions entre ces deux visions (interprétations?) des missions du formateur. Pour autant, les formateurs avouent que ces stratégies «ne produisent rien non plus », ce qui débouche sur une autre tension et un malaise profond: «je le fais pour pas me renier mais je sais que c'est inefficace! » ou encore : « je suis obligé de vendre mais en même temps je les dupe! ». Cette situation est douloureusement vécue par de nombreux formateurs qui déploient une énergie et un temps importants à tenter de compenser ce qui, de leur avis même, n'est pas compensable car soumis à une logique contraire à ce qu'ils croient pertinent pour les étudiants. De fait, nous pouvons, en nous référant à Clot (2010), craindre que la fatigue ainsi engendrée ne soit pas une saine fatigue (celle causée par une activité dans laquelle on se reconnaît et dont on peut atteindre les buts). Nous nous trouvons ici confronté à une véritable perte de sens et de repères professionnels qui confèrent à l'activité des formateurs de l'IUFM un caractère souvent douloureux et lassant. 


\subsection{Un sentiment de déprofessionnalisation}

39 Ces tensions, ces difficultés, ces empêchements exprimés par les formateurs nous ont amené à nous demander si tout cela ne relevait pas d'un processus de déprofessionnalisation ou tout du moins d'un sentiment de déprofessionnalisation (Périsset-Bagnoud, 2010) ? La déprofessionnalisation, telle que nous l'entendons et la définissons, ne peut être assimilée à une simple transformation de la professionnalité d'un groupe fortement déstabilisé par des changements institutionnels mais procède d'une perte d'autonomie et d'un assujettissement aux règles de contrôle (Demailly \& De la Broise, 2009). Comme le soulignent ces auteurs, la déprofessionnalisation est rarement «sèche» dans les faits. Elle est le plus souvent combinée avec des processus de recomposition de nouvelles professionnalités comme par exemple celui de responsabilisation individuelle. Le mouvement de diversification des tâches évoqué plus haut et sous contrôle bureaucratique (les vives réactions à ce que beaucoup appellent le «diktat des logiciels» de contrôle en témoignent) ne serait qu'un prétexte à un mouvement de perte d'autorité de l'acteur dans son rapport au travail et aux autres. En ce sens, il nous semble bien que l'on peut parler ici de sentiment de déprofessionnalisation exprimé par les formateurs de l'IUFM. Ce sentiment est d'autant plus douloureusement vécu que beaucoup d'entre eux se sentent obligés d'user de stratégies de compensation non reconnus par l'institution, de façon autonome, tout en étant par ailleurs soumis à un contrôle bureaucratique très fort de leurs services. Nous pourrions dire que « la réduction normative du métier d'universitaire à un agrégat de missions dont on ne discerne plus le caractère central ou périphérique opère comme une injonction paradoxale à un engagement tous azimuts » Demailly et De La Broise, 2009, p. 5).

Ce sentiment de déprofessionnalisation s'est également exprimé à propos de l'encadrement des mémoires de recherche. La décision initiale de ne confier le suivi de mémoire qu'à une catégorie de formateurs ( $\mathrm{PU}, \mathrm{MCF}$ et docteurs) a été perçue comme une non reconnaissance des compétences que tous les formateurs d'IUFM avaient développées lors de l'encadrement des mémoires professionnels: «avant on était compétent pour encadrer des mémoires, et subitement on ne l'est plus!»Ce sentiment s'est exacerbé lorsque, pour des raisons liées "au défi du nombre", il a été demandé à ces mêmes formateurs d'accepter malgré tout d'encadrer des mémoires.

41 Le sentiment de déprofessionnalisation s'est également exprimé à propos de la difficile acculturation des formateurs de statut second ou premier degré à la culture universitaire. En matière de recherche, certes, mais pas uniquement. Certains des formateurs considèrent par exemple que «les PU et les MCF s'y retrouvent enfin » alors qu'euxmêmes « cherchent à se mettre à niveau !».

42 Pour ces deux derniers éléments (encadrement mémoire, acculturation universitaire) s'agit-il bien d'un processus de déprofessionnalisation au sens que lui donnent Demailly et De la Broise ? Les formateurs sont confrontés à des situations de travail nouvelles pour lesquelles ils n'ont pas forcément toute l'expérience ou les compétences. Ils sont de fait déstabilisés et contraints de s'adapter. On ne peut donc pas parler ici de perte d'autonomie et d'assujettissement à des règles de contrôle. Mais la déstabilisation est forte et la perte de confiance en soi et, dans certains cas de l'institution envers eux (exemple de l'encadrement des mémoires de recherche) est vécue par les acteurs comme 
un véritable processus, sinon de déprofessionnalisation, tout du moins de déqualification ou de disqualification (Paugam, 2009 ; Castel, 2009).

Dans une période de crise institutionnelle comme celle que vivent les IUFM depuis quelques années, l'analyse de ces processus identitaires semble décisive pour la compréhension de l'activité des acteurs en charge de la formation des enseignants au sein des masters.

\section{Discussion.}

Les séminaires organisés visaient trois objectifs principaux ${ }^{15}$. Si le dernier s'inscrit dans une perspective de régulation proactive au sens de la proposition de mise en œuvre de remédiations (aménagement des conditions d'étude et d'enseignement, dispositifs d'accompagnement des formateurs ${ }^{16}$, les deux premiers amènent à envisager ces séminaires comme des moments réflexifs visant l'auto-régulation individuelle et collective de la prise en charge des situations de travail. L'expression par les participants de ce qui les a affectés dans cette réforme et de ce qu'ils disent en avoir fait semblerait néanmoins trouver dans cet exercice même ses limites :

On peut tout d'abord se demander si la perspective compréhensive envisagée dans cette étude et faisant la part belle à la "vérité subjective » des acteurs ne viendrait pas faire obstacle à l'expression d'une "vérité plus objective» visant à mettre au jour des régularités dans le poids des mécanismes institutionnels, structurels et relationnels dans l'évolution de la professionnalité des formateurs. Les différents empêchements ou difficultés évoqués par les formateurs peuvent-ils légitimement documenter l'analyse des conséquences de cette réforme ou sont-ils disqualifiés car trop «subjectifs"? Nous considérons en fait qu'il n'y pas à proprement parler de contradiction mais « une discordance entre ces deux vérités, expressions de deux points de vue, mais avec cette particularité que l'une (la vérité subjective) entre dans l'autre (la vérité objective), ou plus exactement que l'une devrait rentrer dans l'autre pour que cette dernière soit tout à fait complète: la vérité objective, en effet, manquerait à ses propres prétentions si elle n'incluait pas les vérités subjectives, qui font... objectivement partie du monde social» (Lordon, 2013, pp. 79-80). Notre étude, de façon certes très partielle, tente d'inscrire l'expression de la subjectivité des acteurs dans le cadre d'un processus plus large de déterminations institutionnelles et structurelles représentées par cette réforme de la mastérisation et par ses diverses mises en œuvre locales. En cela, ce travail interroge un moment de transition particulier mais qui peut éclairer ces processus dans d'autres contextes et d'autres situations.

Si le dispositif proposé ici se donne pour objectif de permettre et favoriser la régulation institutionnelle et l'auto-régulation individuelle et collective, le niveau d'analyse (celui des discours situés des formateurs) interroge les nombreux développements que nous avons effectué à propos de leur activité. Il s'agit de rester prudent quant aux effets supposés de ce travail de séminaire sur l'activité professionnelle des formateurs. En effet, l'analyse des discours porte sur une activité seconde: celle relative à l'analyse des conséquences de la réforme sur leur activité professionnelle et non sur les effets réels de cette réforme sur leur activité ni sur les effets de ce séminaire sur l'activité professionnelle que nous n'avons pu documenter ici. 
Une troisième limite doit enfin être évoquée : la place particulière qu'occupe le chercheur dans cette étude. Cette étude a été menée dans le cadre d'une mission à la formation de formateurs dans sa propre structure, au sein de laquelle il exerce son activité d'enseignant-chercheur et est lui-même affecté par les changements institutionnels, structurels et relationnels. Pour autant, nous rejoignons Schurmans (2009) et sa position pour une épistémologie compréhensive critiquant la position d'extériorité du chercheur par rapport à son objet: «par sa participation expérientielle à l'interaction sociale, le sujet épistémique perçoit l'objet de l'intérieur. Cela signifie que, quel que soit son objet d'étude et quelles que soient ses techniques méthodologiques, sa démarche, tout à la fois, l'affecte lui-même, affecte autrui et affecte le monde auquel il participe » (Schurmans, 2009, p. 95). Nous nous plaçons ainsi dans la perspective d'une recherche-intervention assumant cette double posture d'extériorité/intériorité.

\section{Conclusion}

En partant de la caractérisation et de l'analyse des situations de travail et de l'analyse par les formateurs eux-mêmes de l'activité qu'ils y déployaient, les séminaires organisés dans les différents sites d'un IUFM visaient avant tout à soigner le travail (Clot, 2010). La situation de transition induite par la réforme met les professionnels des IUFM en position de questionnement et d'incertitude quant au sens de leur métier, aux critères de qualité de leur activité. Or pour améliorer la qualité de vie au travail, ne faut-il pas d'abord se préoccuper de la qualité du travail et la mettre en débat? Les IUFM (et aujourd'hui les ESPE) sont des structures et des institutions constituées d'individus qui,

sans être des sujets libres, sont des pôles d'activité puissantes, déterminés par leurs affects et leurs désirs à faire mouvement d'une certaine manière, le plus souvent pour se conformer aux réquisits de l'institution, mais parfois aussi pour s'en affranchir, voire entrer en guerre contre elle si elle s'est rendue odieuse au point de faire naître le désir de la détruire. (Lordon, 2013, pp. 16-17)

Ces séminaires nous semblent avoir révélé de façon explicite en quoi les formateurs des IUFM balancent entre ces deux tentations: celle de faire leur travail malgré tout ou rejeter ce système dans lequel ils ne se reconnaissent plus. L'institution et les structures ont donc tout intérêt à appréhender cette question dans sa double réalité objective et subjective pour espérer atteindre les objectifs qui sont les siens. Ce dispositif de recherche - intervention est un premier pas dans cette direction.

Castel, R. (2009). La montée des incertitudes. Travail, protections, statut de l'individu. Paris : Seuil, coll. « La couleur des idées».

\section{BIBLIOGRAPHIE}

Altet, M., Paquay, L., \& Perrenoud, P. (2002). Formateurs d'enseignants. Quelle professionnalisation? Bruxelles : De Boeck. 
Amigues, R. (2003). Pour une approche ergonomique de l'activité enseignante. Skholê, hors-série 1, 5-16.

Bardin, L. (1991). L'analyse de contenu. Paris : PUF.

Bourdoncle, R. (1993). La professionnalisation des enseignants : les limites d'un mythe. Revue Française de Pédagogie, 105, 83-119.

Bourdoncle, R. (2007). « Universitarisation », Recherche et formation, 54, 134-145.

Brau-Antony, S. \& Jourdain, C. (2004). Rapport de l'observatoire des formations. Evaluation des formations par les stagiaires du premier et second degré. Rapport de recherche. IUFM Champagne-Ardenne, 103 pages.

Charlot, B. (1990). Enseigner, former : logique des discours constitués et logique des pratiques, Recherche et Formation, 8, 5-18.

Clot, Y. (1999). La fonction psychologique du travail. Paris : PUF.

Clot, Y. (2008). Travail et pouvoir d'agir. Paris : PUF

Clot, Y. (2010). Le travail à cœur. Pour en finir avec les risques psychosociaux. Paris : La Découverte.

Cornu, B. (2015). Teacher Education in France : Universitisation and professionalisation from IUFMs to ESPEs. Education Inquiry, 6(3), 289-307

Demailly, L., \& De la Broise, P. (2009). Les enjeux de la déprofessionnalisation. Études de cas et pistes de travail. Socio-logos, 4, 1-16.

Dubar, C. (1992). Formes identitaires et socialisation professionnelle, Revue Française de sociologie, 4, 505-529.

Ferron, O., Humblot, J.-P., Mayen, P. et al. (2006). Introduire une référentiel de situations dans les référentiels de diplôme en BTS, rapport de recherche de l'unité propre Développement professionnel et formation, département des sciences de la formation et de la communication, Établissement national d'enseignement supérieur agronomique de Dijon.

Lang, V. (2001). Les rhétoriques de la professionnalisation. Recherche et Formation, 38, 95-112.

Leplat, J. (1991). Compétence et ergonomie. Dans R. Amalberti, M. de Montmolin \& J. Theureau, (Dir.), Modèles en analyse du travail (pp. 263-268), Bruxelles : Mardaga.

Lordon, F. (2013). La société des affects. Pour un structuralisme des passions. Paris : Seuil.

Maleyrot, E. (2013). Les professeurs des écoles maîtres formateurs saisis par les épreuves professionnelles. Notes $d u$ CREN $n^{\circ} 16$.

Maroy, C. (2006). Les évolutions du travail enseignant en France et en Europe : facteurs de changement, incidences et résistances dans l'enseignement secondaire. Revue Française de Pédagogie, 155, 111-142.

Mayen, P., Métral, J.F., \& Tourmen, C. (2010). Les situations de travail. Références pour les référentiels, Recherche et Formation, 64, 31-46.

Pastré, P. (2002). L'analyse du travail en didactique professionnelle. Revue française de pédagogie, 138, 9-17.

Pastré, P. (2004). Le rôle des concepts pragmatiques dans la gestion de situations problèmes : le cas des règleurs en plasturgie. Dans R. Samurçay \& P. Pastré (Dir.), Recherches en didactique professionnelle (pp. 17-48), Toulouse, Octarès. 
Paugam, S. (2009). La disqualification sociale. Essai sur la nouvelle pauvreté. Paris : Presses Universitaires de France, coll. « sociologies », dernière édition dans la coll. « Quadrige ».

Perez-Roux, T. (2012). Mutations institutionnelles et remaniements identitaires : enseignants et formateurs face aux réformes, Les Sciences de l'Éducation pour l'Ère Nouvelle, 45(3).

Périsset Bagnoud, D. (2010). Le sentiment de professionnalisation des enseignants et les nouvelles gouvernances : un entre-deux en jachère, Travail et formation en éducation, 7.

Ria, L. (2008). Ergonomie du travail enseignant. Dans A. Van Zanten (Ed.). Dictionnaire de l'Éducation (pp. 282-284), Paris : PUF.

Ricoeur, P. (1990). Soi-même comme un autre, Paris : Le Seuil.

Roger, J.L. (2007). Refaire son métier. Ramonville Saint-Agne : Erès.

Saujat, F. (2002) Ergonomie de l'activité enseignante et développement de l'expérience professionnelle : une approche clinique du travail du professeur. Thèse de Doctorat, 13/12. Aixen-Provence : Université de Provence.

Saujat, F. (2004). Comment les enseignants débutants entrent dans le métier. Formation et pratiques d'enseignement en questions, 1, 97-106.

Schön, D., (1994). Le praticien réflexif. Montréal : Les Éditions Logiques.

Schurmans, M.N. (2009). L'approche compréhensive et qualitative dans la recherche en formation. Education permanente, 177, 91-103.

Schwartz, Y. (2012). Expérience et connaissance du travail ( $3^{\mathrm{e}}$ édition). Paris : Les éditions sociales.

Six, F. (1999). De la prescription à la préparation du travail. Apports de l'ergonomie à la prévention et à l'organisation du travail sur les chantiers du BTP. Habilitation à diriger des recherches, Université Charles de Gaulle, Lille 3.

Vergnaud, G. (1990). La théorie des champs conceptuels. Recherches en didactique des mathématiques, 10, 2-3, 133-170.

Vergnaud, G. (1996). Au fond de l'action, la conceptualisation. Dans J.M. Barbier, Biennale de l'éducation et de la formation, Savoirs théoriques et savoirs d'action (pp. 275-292), Paris : PUF.

Vinatier, I. (2009). Pour une didactique professionnelle de l'enseignement. Rennes : Presses Universitaires de Rennes.

\section{NOTES}

1. Pour des questions de commodité et pour éviter toute ambiguïé nous conserverons tout au long de l'article l'appellation «formateurs» malgré le glissement sémantique vers le terme « enseignants» que nous serons amenés à aborder dans le texte.

2. Pour une présentation détaillée de ces évolutions, voir B. Cornu (2015)

3. L'étude ayant été réalisée au printemps 2012, soit avant la création des ESPE, nous continuerons à parler des formateurs en IUFM.

4. Nous avons pris la liberté de remplacer le terme "enseignant» dans la citation de Saujat par celui de "formateur», considérant que cette assertion était transposable en l'état pour ces derniers.

5. Les épreuves professionnelles conditionnelles déterminent l'accès ou le maintien des acteurs dans la fonction (Maleyrot, 2013). Le statut des formateurs ainsi que le processus de mastérisation posent de fait des questions importantes de ce point de vue. 
«Les épreuves professionnelles situationnelles concernent l'exercice même de la fonction. Elles ne sont pas assujetties à un choix des acteurs (s'engager ou non) mais s'imposent à eux dans leurs situations de travail » (Maleyrot, 2013, p. 3).

6. Nous nous référons ici au sens de l'action de Spinoza rappelé par Lordon (2013, p. 16) : «par rencontre de certaines choses extérieures, les individus subissent certaines affections qui les affectent d'une certaine manière, et les déterminent à désirer faire certaines choses ».

7. Ces onze classes de situations de travail sont les suivantes: faire cours/enseigner/former; suivi individualisé; concertation/harmonisation; organisation de son travail; situations de travail liées à l'activité de recherche; communication externe et promotion de l'institut; se former; évaluation/notation; production de ressources pour la formation; missions particulières (charge de mission, direction d'étude, responsabilités d'UE ou d'EC; engagement volontaire dans la structure/militantisme (élu...).

8. Unité d'Enseignement.

9. Élément Constitutif de l'UE.

10. Au moment de l'écriture de cet article, la formation dite "professionnelle » se faisait après le master dans le cadre d'une année de stage où l'enseignant était à temps plein devant des classes.

11. $\mathrm{PLC} 1=$ professeur de lycée et collège $1^{\text {re }}$ année $; \operatorname{PLC} 2=$ professeur de lycée et collège $2^{\mathrm{e}}$ année $; \mathrm{PE}=$ professeur des écoles $1^{\text {re }}$ année $;$ professeur des écoles $2^{\mathrm{e}}$ année.

12. $\mathrm{M} 1=$ master $1 ; \mathrm{M} 2=$ master 2 .

13. Là encore, le remplacement du terme "stagiaire " par le terme "étudiant » est une illustration de ce déplacement.

14. Élément Constitutif d'une unité d'enseignement (un cours).

15. Réaliser un diagnostic partagé des conséquences de la réforme dite de la « mastérisation » sur la professionnalité des formateurs de l'IUFM Champagne Ardenne ; permettre à tous les acteurs de l'IUFM de s'emparer de ce diagnostic pour définir les possibilités d'un développement du pouvoir d'agir et de l'élargissement de la prise en main personnelle et collective de leur métier (cf. Roger, 2007); donner à la direction et au service de formation continue des éléments susceptibles de les aider à apporter des réponses aux difficultés identifiées (en termes d'organisation du travail, de définition d'espaces spécifiques de réflexion et de travail, d'actions de formation).

16. Des décisions ont été prises par l'équipe de direction et la mission «formation de formateurs » suite à ces séminaires mais après la rédaction initiale de cet article. Nous avons donc fait le choix de ne pas les intégrer pour ne pas allonger le propos.

\section{RÉSUMÉS}

Cet article rend compte d'une étude des conséquences de la mastérisation de la formation des maîtres sur le travail des formateurs d'un IUFM. Elle a tout d'abord un but de diagnostic en vue de l'élaboration d'un plan de formation continue et tente, à partir de l'analyse de situations de travail avec une méthodologie inspirée de la didactique professionnelle (Mayen, Métral \& Tourmen, 2010) et des grilles de lecture de l'activité des professionnels de la clinique de l'activité (Clot, 2008), de mettre au jour la façon dont les enseignants de cet IUFM perçoivent et vivent les conséquences de cette réforme, abordée comme un moment de transition institutionnel et professionnel particulier, sur leur propre professionnalité. Elle vise ensuite, à travers le dispositif 
réflexif support du recueil de données, de créer les conditions de la réappropriation individuelle et collective de leur métier par les formateurs.

This article reports on a study of the consequences of the implementation of a Master Program in Teacher Training on the real work of the teacher trainers from a IUFM (Teacher Training Center). The study first aims at making a diagnosis in order to develop an ongoing training plan/ program. It also attempts to highlight how the teachers from this IUFM view and deal with the consequences of the reform in their proper teaching style, on the basis of the analysis of work situations following a methodology adapted from professional teaching practice (Mayen, Métral \& Tourmen, 2010) as well as reading tables/grids used to analyze professionals' activity in the Clinic of Activity approach (Clot, 2008). The purpose of this study is also to create appropriate conditions to enable teacher trainers to reclaim their own profession, individually and collectively.

INDEX

Mots-clés : réforme, situations de travail, activité, empêchement, identité

Keywords : reform, working situations, activity, hindrance, identity

\section{AUTEUR}

\section{VINCENT GROSSTEPHAN}

Maître de conférences en sciences de l'éducation, CEREP (EA 4692), ESPE de l'académie de Reims 